\title{
MELHORIA DA QUALIDADE NOS SERVIÇOS DE TRANSPORTE UTILIZANDO A FERRAMENTA FMEA
}

\section{QUALITY IMPROVEMENT IN THE TRANSPORT SERVICES USING THE FMEA TOOL}

\author{
Cristiano Roos ${ }^{1}$; Jorge André Ribas Moraes ${ }^{2}$; Leandro Cantorski da Rosa ${ }^{3}$ \\ ${ }^{1}$ Universidade Federal de Santa Maria - UFSM - Santa Maria - Brasil croos@viavale.com.br \\ ${ }^{2}$ Universidade de Santa Cruz do Sul - UNISC - Santa Cruz do Sul - Brasil jorge@unisc.br \\ ${ }^{3}$ Universidade Federal de Santa Maria - UFSM - Santa Maria - Brasil leski78@hotmail.com
}

\begin{abstract}
Resumo
Este trabalho apresenta a ferramenta Failure Modes and Effects Analysis (FMEA) inserida numa abordagem voltada para a melhoria da qualidade nos serviços de transporte. Para isso, realizou-se um estudo de caso em que se implantou a ferramenta numa empresa atuante no segmento de transporte terrestre e aéreo de passageiros e cargas. Com o estudo de caso estabeleceu-se um conjunto de ações que minimizassem ou eliminassem modos de falha em potencial em um dos desdobramentos de serviços prestados pela empresa. O ponto específico em que se realizou a implantação foi na gestão dos pneus dos veículos de transporte terrestre de passageiros, pois falhas relacionadas a este componente geram insatisfações de clientes e aumentam os custos de manutenção na empresa. O objetivo deste trabalho foi verificar, após a implementação das ações, quais as vantagens em se utilizar a ferramenta FMEA como provedora da melhoria contínua da qualidade. Os resultados do trabalho foram alcançados ao se verificar que a FMEA pode ser empregada como instrumento para a melhoria da qualidade e para o aumento da confiabilidade $e$ segurança dos serviços prestados pela empresa de transportes estudada. Deste modo, a realização do presente estudo de caso proporcionou um maior entendimento acerca da temática proposta, além de mostrar a importância da melhoria contínua na gestão da qualidade.
\end{abstract}

Palavras-chave: gestão da qualidade, melhoria contínua, FMEA.

\section{Introdução}

A busca constante das organizações brasileiras pela melhoria da qualidade está se consolidando como uma das estratégias competitivas mais seguidas nos diferentes segmentos de mercado. Esta realidade é motivada pela vantagem competitiva que a qualidade proporciona frente as crescentes exigências dos consumidores. Eckes (2001, p.15), em uma de suas citações afirma que “apesar do enfoque em formas inovadoras de criar produtos e prestar serviços, uma constante permanece: as empresas que oferecem produtos e serviços de melhor qualidade sempre vencem a 
concorrência”. Segundo Karsak et al. (2002), a competitividade globalizada estimula as empresas a buscarem níveis mais altos de qualidade para seus produtos e serviços.

Neste sentido, desenvolveu-se neste trabalho uma abordagem utilizando a ferramenta FMEA (Failure Modes and Effects Analysis) como instrumento para a melhoria da gestão da qualidade na prestação de serviços. Para desenvolver esta abordagem realizou-se um estudo de caso em que se implantou a ferramenta numa empresa prestadora de serviços atuante no segmento de transporte terrestre e aéreo de passageiros e cargas. O objetivo principal do trabalho foi verificar a viabilidade de utilização da ferramenta FMEA como provedora da melhoria contínua da qualidade nesta empresa, bem como balizar futuros trabalhos científicos neste contexto.

Conforme os dados da empresa, esta conta com o trabalho de 1080 funcionários e com uma frota composta de 251 ônibus, 16 caminhões, 14 veículos utilitários e 2 aeronaves. Apresenta números bastante significativos (média mensal): transporte de 366 mil passageiros, transporte de 2,12 mil toneladas de carga, 3,06 milhões de quilômetros rodados e 600 horas de vôo.

Relatos da empresa identificavam como ponto crítico global na prestação dos serviços, o elevado índice de falhas relacionadas aos pneus dos veículos de transporte de passageiros. Deste modo, buscou-se, com a ferramenta FMEA, identificar a melhor forma de aplicação e utilização dos pneus, a fim de aumentar a confiabilidade e segurança do sistema em que estão inseridos e conseqüentemente interferir positivamente na qualidade dos serviços de transporte prestados.

\subsection{Gestão da qualidade}

Para Brocka e Brocka (1994, p. 4), de um modo simplificado, a gestão da qualidade é definida como sendo: "melhorias sistemáticas e contínuas na qualidade dos produtos, serviços e na vida das pessoas, utilizando todos os recursos humanos e financeiros disponíveis"; "uma metodologia de resolução de problemas e aperfeiçoamento de processos sobre toda a empresa"; e "um sistema de meios para economicamente produzir bens ou serviços que satisfaçam as necessidades dos clientes”. Já para Paladini (2006), a qualidade assume um conceito que envolve múltiplos elementos, com diferentes níveis de importância no decorrer do tempo, tornando assim a definição de gestão da qualidade dinâmica.

\subsection{Melhoria contínua da qualidade}

A melhoria contínua da qualidade é parte integrante da maioria dos sistemas de gestão da qualidade. Visa aumentar continuamente a eficiência e eficácia da gestão da qualidade nas organizações (ISO/TS 16949, 2002; NBR ISO 9001, 2000). O conceito de melhoria contínua sugere 
um processo sem fim, sendo que a maneira mais usual de realizar esta melhoria é com o auxílio do ciclo PDCA (ciclo planejar, fazer, checar e agir), seqüência de etapas de solução de problemas que são vistas como operacionalizando um ciclo (SIMÕES E ALLIPRANDINI, 2006).

\subsection{Pneu}

Nos veículos modernos, exceto aos que se referem às forças aerodinâmicas, todas as forças atuantes que determinam como o veículo efetua curvas, freia e acelera, são gerados numa pequena área de contato entre o solo e os pneus (GILLESPIE, 1992). Em mais de cem anos desde a invenção do pneu, várias idéias alternativas vêm sendo investigadas, e algumas até mesmo testadas em pista. Entretanto não houve aplicação comercial para nenhuma delas, permanecendo o domínio do pneumático de borracha, resultado da combinação de propriedades que permitem ao pneu oferecer suporte e controle do veículo com boa durabilidade em diferentes condições de uso (DIXON, 1996).

\subsection{Ferramenta FMEA}

Para os autores Stamatis (2003) e Helman (1995), a FMEA é uma ferramenta utilizada para identificar, minimizar e eliminar falhas conhecidas ou potenciais, de sistemas, processos, projetos, produtos e prestação de serviços, antes que estas atinjam o cliente e a empresa. Já para Palady (2004, p.5), a FMEA é uma metodologia que oferece três funções distintas, que são: "FMEA é uma ferramenta para prognóstico de problemas"; "FMEA é um procedimento para desenvolvimento e execução de projetos, processos ou serviços, novos ou revisados"; e "FMEA é o diário do projeto, processo ou serviço".

Puente et al. (2002), afirma que o primeiro método associado àquilo que a ferramenta FMEA propõe foi inicialmente utilizado pela National Aeronautics and Space Administration (NASA) em 1963; e então expandido para a indústria automobilística, onde foi utilizada para detectar, quantificar e ordenar possíveis defeitos potenciais no estágio de projeto de produtos, antes de chegarem ao consumidor final.

Segundo Moretti e Bigatto (2006, p.2), ao final da década de 80, "através de uma força de trabalho composta por representantes da Chrysler Corporation, Ford Motor Company e General Motors Corporation desenvolveu-se a norma QS 9000, em que foi incluído a FMEA como uma das ferramentas de planejamento avançado da qualidade. Em fevereiro de 1993, a AIAG (Automotive Industry Action Group) e a ASQC (American Society for Quality Control) patentearam os padrões relacionados ao FMEA, criando um manual. O mesmo vale para a SAE (Society of Automotive Engineers) detentora do procedimento SAE J-1739 que trata da FMEA". 
Diversas aplicações da ferramenta FMEA têm sido relatadas por pesquisadores de diversas áreas: como suporte ao gerenciamento de riscos em serviços hospitalares (PAPARELLA, 2007; REID, 2005); avaliação de riscos associados à estratégia de terceirização (WELBORN, 2007); análise de falhas existentes em etapas de sistemas agroindustriais (GARRAFA, 2005; GARRAFA e ROSA, 2004).

Segundo Puente et al. (2002), a ferramenta FMEA é desenvolvida basicamente em dois grandes estágios. No primeiro estágio, possíveis modos de falhas de um sistema, processo, projeto, produto ou serviço e suas respectivas causas e efeitos são identificados. No segundo estágio, é determinado o nível crítico, isto é, a pontuação de risco destas falhas. As falhas mais críticas serão as primeiras do ranking, e serão consideradas prioritárias para a aplicação de ações de melhoria.

Leal, Pinho e Almeida (2006), explicam que há três índices utilizados na ferramenta FMEA para a definição das prioridades das falhas, quais sejam: índice de ocorrência, índice de severidade e índice de detecção. "A ocorrência define a freqüência da falha, enquanto a severidade corresponde à gravidade do efeito da falha. A detecção é a habilidade para detectar a falha antes que ela atinja o cliente" (LEAL, PINHO e ALMEIDA, 2006). Utilizando a metodologia tradicional, a multiplicação destes três índices, que possuem escalas de 1 a 10, vai resultar no Risk Priority Number (RPN), que será responsável pelo ranking das falhas (AGQ, 2006; PALADY, 2004). As Figuras 1 a 3 apresentam a classificação dos três índices utilizada na realização deste trabalho.

A forma de apresentação da ferramenta FMEA é no formato de formulários físicos ou digitais. Nestes formulários reúnem-se todas as informações relevantes da ferramenta para facilitar no seu desenvolvimento, análise e interpretação (AGQ, 2006).

Os estudos de Palady (2004) demonstram que a ferramenta FMEA é mais eficaz quando aplicada por uma equipe. Para ele, quando se reúne o conhecimento coletivo de todos da equipe, se tem um resultado ou retorno significativo de qualidade e confiabilidade. Assim, ainda para o mesmo autor, essa equipe deve ser formada por um grupo de quatro a sete pessoas que compreendam como o sistema, processo, produto ou serviço é projetado, produzido, utilizado e mal utilizado.

Figura 1 - Escala de severidade

\begin{tabular}{|l|c|}
\hline Escala de severidade dos efeitos dos modos de falha & Índice de severidade \\
\hline Efeito não percebido pelo cliente & 1 \\
\hline Efeito bastante insignificante, percebido por $25 \%$ dos clientes & 2 \\
\hline Efeito insignificante, mas percebido por $50 \%$ dos clientes & 3 \\
\hline Efeito moderado e percebido por $75 \%$ dos clientes & 4 \\
\hline Efeito consideravelmente crítico, percebido pelo cliente & 5 \\
\hline Efeito consideravelmente crítico, que perturba o cliente & 6 \\
\hline Efeito crítico, que deixa o cliente um pouco insatisfeito & 7 \\
\hline Efeito crítico, que deixa o cliente consideravelmente insatisfeito & 8 \\
\hline Efeito crítico, que deixa o cliente totalmente insatisfeito & 9 \\
\hline Efeito perigoso, que ameaça a vida do cliente & 10 \\
\hline
\end{tabular}

Fonte: Adaptado de Palady, 2004 
Figura 2 - Escala de ocorrência

\begin{tabular}{|l|c|}
\hline Escala de avaliação da ocorrência das causas e modos de falha & Índice de ocorrência \\
\hline Extremamente remoto, altamente improvável & 1 \\
\hline Remoto, improvável & 2 \\
\hline Pequena chance de ocorrência & 3 \\
\hline Pequeno número de ocorrências & 4 \\
\hline Espera-se um número ocasional de falhas & 5 \\
\hline Ocorrência moderada & 6 \\
\hline Ocorrência freqüente & 7 \\
\hline Ocorrência elevada & 8 \\
\hline Ocorrência muito elevada & 9 \\
\hline Ocorrência certa & 10 \\
\hline
\end{tabular}

Fonte: Palady, 2004

Figura 3 - Escala de detecção

\begin{tabular}{|l|c|}
\hline Escala de detecção das causas e modos de falha & Índice de detecção \\
\hline É quase certo que será detectado & 1 \\
\hline Probabilidade muito alta de detecção & 2 \\
\hline Alta probabilidade de detecção & 3 \\
\hline Chance moderada de detecção & 4 \\
\hline Chance média de detecção & 5 \\
\hline Alguma probabilidade de detecção & 6 \\
\hline Baixa probabilidade de detecção & 7 \\
\hline Probabilidade muito baixa de detecção & 8 \\
\hline Probabilidade remota de detecção & 9 \\
\hline Detecção quase impossível & 10 \\
\hline
\end{tabular}

Fonte: Palady, 2004

\section{Metodologia}

Para o desenvolvimento deste trabalho realizou-se um estudo de caso com abordagem qualitativa e com complementação de dados quantitativos. Segundo Gil (2002, p. 54), um estudo de caso é caracterizado pelo "estudo profundo e exaustivo de um ou poucos objetos, de maneira que permita seu amplo e detalhado conhecimento". Para Salomon (2001), enquanto os dados quantitativos de uma pesquisa são utilizados para descrever uma variável quanto a sua tendência central e sua freqüência, os dados qualitativos são basicamente úteis para quem busca entender o contexto onde algum fenômeno ocorre.

O estudo de caso realizado teve seu marco inicial numa necessidade encontrada na metodologia de melhoria contínua da qualidade praticada na empresa de transportes. Isto é, a partir de uma análise dos pontos críticos na prestação dos serviços, identificou-se uma necessidade que se transformou numa oportunidade para o desenvolvimento de uma abordagem voltada para a melhoria da qualidade utilizando-se a ferramenta FMEA. Partiu-se então para a definição de quais seriam os objetivos almejados e implantou-se a ferramenta. Após a implantação avaliou-se os resultados da abordagem sob o ponto de vista científico e do cliente. Para Dikmen et al. (2004), o sucesso de uma organização depende principalmente de como ela utiliza seus recursos para satisfazer as necessidades dos clientes. 


\section{Implantação da Abordagem}

A implantação da ferramenta FMEA, dentro do contexto da abordagem voltada para a melhoria da qualidade, foi iniciada com o seu planejamento e suas respectivas definições, como por exemplo, as pessoas da equipe, tempo necessário para as reuniões, recursos financeiros e o escopo principal da implantação. Nesta etapa desprendeu-se uma atenção redobrada, pois conforme Palady (2004), o planejamento detalhado do desenvolvimento da ferramenta FMEA, antes da elaboração e implantação do formulário, garantirá sua eficácia.

Realizou-se a implantação com a colaboração de uma equipe composta de cinco pessoas de diferentes áreas e níveis hierárquicos desta empresa: um gerente do departamento de engenharia, um supervisor do departamento de manutenção, um supervisor do departamento de qualidade, um colaborador do departamento de borracharia e um estagiário do departamento de engenharia. $\mathrm{O}$ tempo empregado para a implantação foi de aproximadamente um semestre, com pelo menos uma reunião semanal. O escopo principal da implantação da ferramenta foi o melhoramento do gerenciamento de falhas dos pneus dos veículos de transporte terrestre de passageiros, pois modos de falha relacionados a este componente tendem a gerar insatisfações de clientes e aumentam os custos de manutenção na empresa.

$\mathrm{Na}$ etapa seguinte iniciou-se o preenchimento dos formulários. Foram elaborados cinco formulários FMEA, um para cada uma das funções conforme definidas na Figura 4. A Figura 4 apresenta as funções definidas pela equipe em seções de brainstorming, para os pneus dos veículos de transporte de passageiros. A Figura 6 apresenta o formulário completo da ferramenta FMEA referente à função de número 3 (não estourar). Vale destacar que os conteúdos dos demais campos do formulário também foram gerados a partir de sessões de brainstorming.

Figura 4 - Funções definidas para os pneus dos veículos de transporte de passageiros da empresa

\begin{tabular}{|l|c|}
\hline Funções definidas & Número da função \\
\hline Não murchar & 1 \\
\hline Não furar & 2 \\
\hline Não estourar & 3 \\
\hline Amortecer parte das irregularidades do solo & 4 \\
\hline Rodar no mínimo o tempo estipulado pelo fabricante & 5 \\
\hline
\end{tabular}

Os modos de falha dos pneus foram definidos pela equipe, levando em consideração a recomendação de Palady (2004) para o preenchimento deste campo, ou seja, o modo de falha é quase na totalidade dos casos a sentença contrária da função. Os efeitos dos modos de falha sob a perspectiva do cliente foram determinados na seqüência da implantação. Foi observado para descrição dos efeitos dos modos de falha novamente uma recomendação de Palady (2004, p.60), 
isto é, "sempre que possível, ao descrever os efeitos resultantes de um modo de falha, a descrição deve refletir a experiência dos clientes através dos sentidos, [...] isso minimizará o risco de subestimar a severidade do efeito".

A seguir, preencheu-se o campo do formulário que se refere à severidade dos efeitos dos modos de falha. Para isto, a equipe baseou-se na escala de severidade apresentada na Figura $1 . \mathrm{Na}$ etapa seguinte, considerada a mais difícil, identificaram-se as causas dos modos de falha. Utilizouse um grande número de dados quantitativos e qualitativos, que foram interpretados com o auxílio do gráfico de Pareto e do diagrama de Ishikawa (RAMOS, 1995; WERKEMA, 1997).

Na seqüência do preenchimento do formulário, a equipe quantificou a ocorrência das causas. Para isto, baseou-se na escala de ocorrência apresentada na Figura 2. Com esta etapa concluída, partiu-se para o preenchimento das colunas correspondentes a "formas de controle" e "detecção". $\mathrm{Na}$ coluna "formas de controle" apresentaram-se as maneiras que a empresa possui para detectar as falhas antes que estas atinjam o cliente, e na coluna "detecção" a equipe estimou com base na Figura 3 a probabilidade de se detectar as causas e modos de falha antes que atinjam o cliente.

Para determinar as prioridades, optou-se pelo método tradicional de interpretação da FMEA, o método RPN, obtido pela multiplicação da pontuação dada para os índices de severidade, ocorrência e detecção. Têm-se assim valores situados numa faixa crescente, que indicam desde baixíssimo risco até risco crítico para o resultado mais elevado. A equipe então estipulou que primeiramente seriam determinadas ações de correção e de minimização dos modos de falha para os RPN's cujos índices fossem iguais ou superiores a 120.

A etapa final consistiu na recomendação das ações para minimizar e solucionar os modos de falha em potencial. Nesta etapa, a mais importante, obteve-se os resultados esperados pela equipe de implantação da FMEA, pois foi a partir destes resultados que se partiu para o efetivo aumento de confiabilidade do sistema. Para isso, foram estabelecidos o limite temporal para implementação destas ações bem como seus respectivos responsáveis. Cabe frisar que as ações recomendadas referem-se principalmente ao treinamento das pessoas envolvidas com a manutenção e utilização dos pneus, Figura 5.

Figura 5 - Porcentagem e natureza das ações de melhoria recomendadas

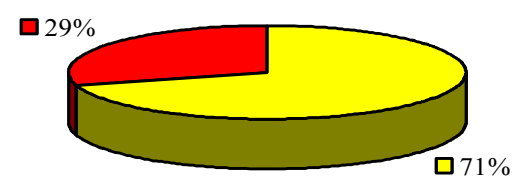

$\square$ Ações de melhoria relacionadas às Pessoas $-71 \%$

$\square$ Ações de melhoria relacionadas aos Materiais - 29\% 
Figura 6 - Formulário FMEA desenvolvido referente à função "Não Estourar"

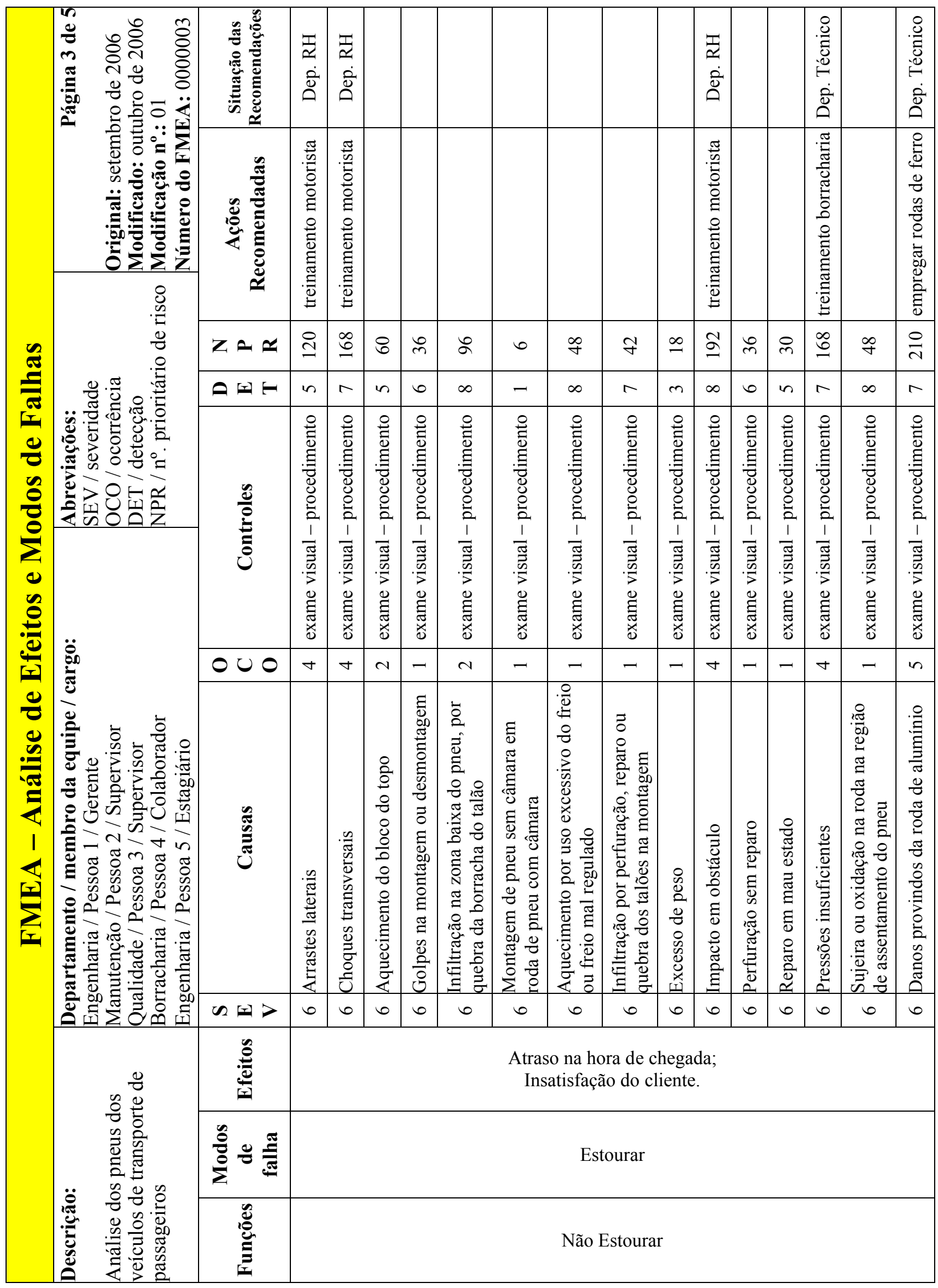




\section{Resultados e Discussões}

O processo de implementação do conjunto de ações, determinadas na etapa final de desenvolvimento da ferramenta FMEA, foi sistemático. Como a maioria das ações de melhoria estavam relacionadas ao treinamento das pessoas envolvidas, Figura 6, estas foram implementadas mediante uma sistemática de capacitação dos trabalhadores. Esta sistemática englobou quatro etapas que foram trabalhadas na seguinte ordem: aprendizado teórico, aprendizado comportamental, aprendizado simulado e aprendizado prático. Cada ciclo de capacitação levou um mês. Desde o início de sua implementação, já era possível visualizar melhorias na gestão de falhas relacionadas aos pneus e na atitude das pessoas comprometidas. Os resultados dos treinamentos, baseados em dados mensuráveis, foram avaliados após a conclusão das três primeiras etapas de aprendizado. Os resultados foram confrontados com os dados históricos da empresa. A diminuição de falhas relacionadas à errônea utilização e manutenção dos pneus pôde ser percebida claramente, pois registrou-se uma redução deste tipo de falha em $12 \%$ até o final desta pesquisa.

Os resultados finais da abordagem voltada para a melhoria da qualidade, utilizando a ferramenta FMEA como provedora, foram bastante positivos. Contudo os resultados no âmbito comportamental das pessoas merecem um lugar de destaque, pois, de certa forma, foi surpreendente o comprometimento e vontade das pessoas em melhorar ainda mais a qualidade dos serviços de transporte prestados pela empresa.

\section{Considerações Finais}

A realização deste trabalho proporcionou um maior entendimento acerca da ferramenta FMEA inserida numa abordagem de melhoria contínua da qualidade nos serviços de transporte terrestre de passageiros. Isto é, determinaram-se e implementaram-se ações de melhoria que vão ao encontro dos resultados almejados pela empresa no contexto da gestão da qualidade, isto é, o aumento de confiabilidade, segurança e qualidade do serviço prestado.

O estudo revelou ainda bons resultados na adequada formação da equipe de trabalho para o desenvolvimento da ferramenta, pois esta foi marcada pela amplitude de alcance, envolvendo quatro departamentos da empresa. Neste sentido, um ponto importante observado após o término da pesquisa foi quanto à percepção de caráter satisfatório dos envolvidos em prevenir, minimizar e eliminar problemas utilizando a FMEA.

Desta forma, do ponto de vista empresarial, mais do que o retorno direto decorrente da minimização e eliminação de falhas potenciais, a empresa estudada teve como benefício com a implantação da FMEA inserida no contexto de melhoria da qualidade, o aumento de confiabilidade 
e segurança do serviço prestado, proporcionando maior satisfação do usuário do serviço de transporte. Já do ponto de vista da satisfação do ser humano, o trabalho desenvolvido possibilitou ganhos no campo motivacional e comportamental, com o resgate de valores no sentido da colaboração e comprometimento das pessoas, que, juntas, podem desenvolver uma atividade em prol do futuro da organização e de si mesmas.

Concluiu-se que a ferramenta FMEA é eficiente e eficaz quando utilizada como abordagem de melhoria contínua da qualidade, visto que os objetivos do trabalho foram alcançados já em curto prazo. A abordagem proposta e desdobrada neste trabalho mediante um estudo de caso, reflete sobre a utilização de novas práticas para o melhoramento contínuo da qualidade e baliza a realização de futuros trabalhos científicos neste contexto.

\begin{abstract}
This paper presents the tool Failure Modes and Effects Analysis (FMEA) inserted in a context gone back to the quality improvement in the transport services. For that, tooked place a case in that the tool was applied in a company that acts on the land/air transportation of passengers and carrier. With the case study was settled down using the tool, actions that they minimized or they eliminated flaw manners in potential in one of the unfoldings of services rendered by the company. The specific point in that took place the was implanted it went in to administration of the tires of the vehicles of passengers' terrestrial transport, because you fail related to this component they generated dissatisfactions to the customers and they increase the costs of maintenance of the company. Thus, the objective of this work went verify, after the implant of the actions, which the advantages in using the tool FMEA as supplying of the continuous quality improvement. The results of the work were reached when verifying that FMEA can be used as instrument for the improvement of the quality and for the increase of the reliability and safety of the services rendered by the studied company of transports. This way, the accomplishment of the present case study provided a larger understanding concerning the thematic proposal, besides showing the importance of the continuous improvement of the administration of the quality in the current days.
\end{abstract}

Key-words: quality management, continuous improvement, FMEA.

\title{
Referências
}

AGQ - ASSOCIAÇÃO GAÚCHA PARA A QUALIDADE. Curso FMEA: Análise de modo e Efeitos de Falha em Potencial. 3. ed. Novo Hamburgo: 2006, 48 p.

BROCKA, B.; BROCKA, M. S. Gerenciamento da Qualidade. Tradução Valdênio Ortiz de Souza. São Paulo: Makron Books, 1994.

DIKMEN, I.; BIRGONUL, M. T.; KIZILTAS, S. Strategic use of quality function deployment (QFD) in the construction industry. Building and Environment, v. 40, n.2, p. 245-255, 2004.

cross'

ECKES, G. A Revolução Seis Sigma. Rio de Janeiro: Campus, 2001.

GARRAFA, M. Aplicação de FMEA na otimização dos fatores de produção da canola. Santa Maria: UFSM, 2005. 166 p. Dissertação (Mestrado em Engenharia de Produção) Universidade Federal de Santa Maria, 2005. 
GARRAFA, M, ROSA, L. C. Priorização de ações em cultivo de canola (Brassica napus L. var.oleifera) na EscolaFazenda da SETREM - subprocesso semeadura. In: XXIV Encontro Nacional de Engenharia de Produção, 2004, Florianópolis. Anais [CD-Rom], 2004.

GIL, A. C. Como elaborar projetos de pesquisa. 3. ed. São Paulo: Atlas, 2002.

HELMAN, H. Análise de falhas (Aplicação dos métodos de FMEA e FTA). Belo Horizonte: Fundação Cristiano Ottoni, Escola de Engenharia da UFMG, 1995.

ISO/TS 16949. The Quality System Requirements for the Design/Development, Production, Installation and Servicing of Automotive Related Products. ABNT, 2002.

KARSAK, E. E.; SOZER, S.; ALPTEKIN, E. Product planning in quality function deployment using a combined analytic network process and goal programming approach. Computers \& Industrial Engineering. Vol. 44, n. 1, p. 171-190, 2002.

cross ${ }^{\text {ref }}$

LEAL, F.; PINHO, A. F.; ALMEIDA, D. A. Análise das falhas através da aplicação do FMEA e da Teoria Grey. Revista Gestão Industrial, Ponta Grossa, n.01, v.02, p. 79-88, jan/mar, 2006.

MORETTI, D. de C.; BIGATTO, B. V. Aplicação do FMEA: estudo de caso em uma empresa do setor de transporte de cargas. Disponível em: <http://www.nortegubisian.com.br/ artigos/fmea.pdf>. Acesso em: 10 jun. 2006.

NBR ISO 9001. Sistemas de Gestão da Qualidade - Requisitos, São Paulo: ABNT, 2000.

PALADINI, E. P. Gestão da qualidade: teoria e prática. 2 ed. São Paulo: Atlas, 2006.

PALADY, P. FMEA: Análise dos Modos de Falha e Efeitos: prevendo e prevenindo problemas antes que ocorram. 3. ed. São Paulo: IMAM, 2004.

PAPARELLA, S. Failure mode and effects analysis: a useful tool for risk identification and injury prevention. Journal of Emergency Nursing, v. 33, n. 4, p. 367-371, 2007.

cross ${ }^{\text {ref }}$

PUENTE, J.; PINO, R.; PRIORE, P. \& LA FUENTE, D. de. A decision support system for applying failure mode and effects analysis. International Journal of Quality \& Reliability Management, n. 2, v. 19, 2002.

cross ${ }^{\text {ref }}$

RAMOS, A. W. Controle Estatístico do Processo. São Paulo: Fundação Carlos Alberto Vanzolini, 1997.

REID, R. D. FMEA - Something old, something new. Quality Progress, v. 38, n. 5, p. 90-93, 2005.

SALAMON, D. V. Como fazer uma monografia. 10. ed. São Paulo: Martins Fontes, 2001.

SIMÕES, R.; ALLIPRANDINI, D. H. Gestão da melhoria contínua: modelo de boas práticas e aplicação em uma empresa de médio porte. In: ENCONTRO NACIONAL DE ENGENHARIA DE PRODUÇÃO, XXVI, 2006. Anais: Fortaleza, 2006.

STAMATIS, D. H. Failure Mode and Effect Analysis: FMEA fron theory to execution. 2. ed. Milwaukee, Winsconsin: ASQ Quality Press, 2003.

WELBORN, C. Using FMEA to assess outsourcing risk. Quality Progress, v. 40, n. 8, p. 17-21, 2007.

WERKEMA, M. C. C. Ferramentas estáticas básicas para o gerenciamento de processos. Belo Horizonte: Fundação Cristiano Ottoni, Escola de Engenharia da UFMG, 1995.

\section{Dados dos Autores:}

Nome completo: Cristiano Roos 
Filiação institucional: Universidade Federal de Santa Maria - UFSM

Departamento: Programa de Pós-Graduação em Engenharia de Produção - PPGEP

Função ou cargo ocupado: Aluno de Mestrando

Endereço completo para correspondência: Av. Léo Kraether, 901, casa 51, bairro Country, Santa

Cruz do Sul, Rio Grande do Sul, Brasil, CEP 96820790

Telefones para contato: (51) 37177212 e (51) 99937456

e-mail:croos@viavale.com.br

Nome completo: Jorge André Ribas Moraes

Filiação institucional: Universidade de Santa Cruz do Sul

Departamento: Engenharia, Arquitetura e Ciências Agrárias

Função ou cargo ocupado: Chefe de Departamento

Endereço completo para correspondência: Av. Independência, 2293, sala 5220, bairro Universitário, Santa Cruz do Sul, Rio Grande do Sul, Brasil, CEP 96815900

Telefones para contato: (51) 37177382

e-mail:jorge@unisc.br

Nome completo: Leandro Cantorski da Rosa

Filiação institucional: Universidade Federal de Santa Maria

Departamento: Engenharia de Produção e Sistemas

Função ou cargo ocupado: Professor no Programa de Pós-Graduação em Engenharia de Produção

Endereço completo para correspondência: Departamento de Engenharia de Produção e Sistemas,

Centro de Tecnologia, UFSM, Campus Camobi, bairro Camobi, Santa Maria, Rio Grande do Sul,

Brasil, CEP 97105900

Telefones para contato: (55) 32208442

e-mail:leski78@hotmail.com

Recebido para publicação em: 11/02/2008

Aceito para publicação em: 06/03/2008 